\title{
The Influence of Behavioral and Psychological Symptoms of Dementia on the Autonomic Nervous System
}

\author{
Daisuke Kimura ${ }^{1}$, Ken Nakatani ${ }^{1}$, Masako Notoya ${ }^{2}$, Aiko Imai ${ }^{3}$, Taro Ohtoshi ${ }^{1}$ \\ ${ }^{1}$ Department of Rehabilitation Sciences, Faculty of Allied Health Sciences, Kansai University of Welfare Sciences, Osaka, Japan \\ ${ }^{2}$ Department of Speech and Hearing Sciences and Disorders, Kyoto Gakuen University, Kyoto, Japan \\ ${ }^{3}$ Division of Occupational Therapy, Faculty of Care and Rehabilitation, Seijoh University, Tōkai, Japan \\ Email: dkimura@tamateyama.ac.jp, knakatani@tamateyama.ac.jo, notoya@kyotogakuen.ac.jp,imai-a@seijoh-u.ac.jp, \\ ohtoshi@tamateyama.ac.jp
}

How to cite this paper: Kimura, D., Nakatani, K., Notoya, M., Imai, A. and Ohtoshi, T. (2017) The Influence of Behavioral and Psychological Symptoms of Dementia on the Autonomic Nervous System. World Journal of Neuroscience, 7, 383-390. https://doi.org/10.4236/wjns.2017.74030

Received: October 25, 2017

Accepted: November 20, 2017

Published: November 23, 2017

Copyright (c) 2017 by authors and Scientific Research Publishing Inc. This work is licensed under the Creative Commons Attribution International License (CC BY 4.0).

http://creativecommons.org/licenses/by/4.0/

\begin{abstract}
Stress is one of the most potent of many factors that can cause expression of behavioral and psychological symptoms of dementia (BPSD). Stress can also increase autonomic nervous activity. This study aimed to examine the relationships between BPSD and autonomic function in the elderly with dementia. We recruited 35 participants diagnosed with dementia from nearby geriatric health service facilities. The Neuropsychiatric Inventory was used for the evaluation of BPSD, and a TAS 9 VIEW (manufactured by YKC) was used for the heart rate variability analysis to measure autonomic nervous system activity. We constructed a model in which the autonomic nervous system comprised a category and performed a structural equation modeling analysis to calculate the standardization coefficient and correlation coefficient for each factor. The results showed that the influence of BPSD resulted in suppression of sympathetic and enhancement of parasympathetic nervous system activity.
\end{abstract}

\section{Keywords}

BPSD, Autonomic Nervous System Activity, Structural Equation Modeling Analysis

\section{Introduction}

Various factors can cause behavioral and psychological symptoms of dementia (BPSD) [1]. Of them, experiencing stress likely results in BPSD in patients with dementia [2].

Human stress-coping mechanisms increase stress responses mediated through 
the sympathetic nervous-adrenal medullary (SAM type) and the hypothalamicpituitary-adrenocortical (HPA type) systems [3]. When dementia becomes stressful, HPA system activity is enhanced, and BPSD develop [4]. Therefore, in dementia, the HPA system and SAM systems can function as indices to better understand BPSD.

If a patient with dementia is coping with stress, the autonomic nervous system becomes dominated by SAM activity. Meanwhile, organic dystrophic changes have been reported in dementia in which neurofibrillary tangles frequently occur in the hypothalamus, the subcortical forebrain region that provides central regulation of the autonomic nervous system [5] [6]. Brain dysfunction due to dementia progression is said to cause autonomic nervous system dysfunction [5] [6]. If stress is a factor in BPSD expression, autonomic nervous system activity is generally sympathetic-dominant. However, assuming that autonomic nervous function is impaired by pathological changes in the brain when a patient with dementia is exposed to stress, it is unclear whether autonomic nervous system activity will become sympathetic-dominantas as it does in a healthy person, or if autonomic nervous system activity will become parasympathetic-dominant instead. This is particularly true when autonomic nervous system activity in dementia is in a state in which BPSD are expressed. If autonomic nervous system activity during BPSD expression can be clarified, it may be possible to predict the appearance of BPSD beforehand. However, there is no prior research that has clarified this issue.

Therefore, this study aimed to examine the relationship between autonomic function and BPSD in the elderly with dementia.

\section{Methods}

\subsection{Participants}

Elderly patients who were diagnosed with dementia in geriatric health service facilities were recruited. The exclusion criteria were those with a history of cerebrovascular disorder, those with a history of depression, and those who have difficulty communicating.

\subsection{Ethics}

In the present study, prior to implementation of the evaluation, a document explaining the purpose of the evaluation was distributed to each subject. After an explanation, subjects who agreed to participate signed the agreement on the consent form. With these procedures for the participants, informed consent was carefully confirmed. The study was conducted in accordance with the ethical standards set forth in the Helsinki Declaration (1983). The entire study protocol was approved by the Kansai University of Welfare Sciences Expert Committee on University Research Ethical Evaluation (approval number 1604).

\subsection{Procedures}

The Neuropsychiatric Inventory (NPI) [7] was used for the evaluation of BPSD. 
A TAS 9 VIEW (YKC, Tokyo, Japan) was used to measure heart rate variability [8] as an index of autonomic nervous system activity. Total power (TP) was used for the evaluation of overall autonomic nervous system activity, with very low frequency (VLF) power used for the evaluation of sympathetic nervous system activity. Low frequency (LF) power was used for the evaluation of the sympathetic/parasympathetic nervous system activity ratio, and high frequency (HF) power was used for the evaluation of parasympathetic nervous system activity. The LF/HF ratio was used for the evaluation of BPSD. The measured values were expressed in natural logarithm (Ln).

Evaluations and measurements were carried out by two occupational therapists. Both occupational therapists had experience in evaluating the NPI and carried out measurements using the TAS 9 VIEW after training, in which they repeated the procedure until the operation of the instrument was smooth.

The environmental conditions for autonomic nervous system measurement were: 1 ) the air conditioner was set to $28^{\circ} \mathrm{C} ; 2$ ) the lighting was set at the reference illuminance of the Japanese Industrial Standards (JIS) standard general rule; 3 ) the sound environment was less than the standard value at the medical care facility; and 4) odors were not uncomfortable for those taking the measurements. The measurement conditions and procedures were as follows: 1) the procedure was performed during the daytime $(13: 00) ; 2)$ only the subject and the occupational therapist were in the room; 3 ) the patients rested in a sitting position (closed eyes); and 4) a pulse wave sensor was used for 2 to 3 minutes for acclimatization and relaxation [9]. The measurement was then carried out for 2 minutes and 30 seconds [9]. Environmental conditions, measurement conditions, and procedures were carried out under the same conditions for all subjects.

\subsection{Data Analysis}

The statistical analysis was conducted according to the following three-stage approach. First, a factor analysis was conducted to identify groupings within each category of lower items on the NPI. To detect clusters of 10 factors, item scores were subjected to a conventional factor analysis using the maximum likelihood with promax rotation method. A factor analysis is a constructed validity tool that aims to identify underlying clinical dimensions. The validity of a symptom cluster has been defined as the common variance of the factor and the construct validity is studied by comparisons with other constructs [10]. Factors with an eigen value exceeding 1.0 and an interpretable constellation of items are usually considered of interest in the clinical description. Secondly, we constructed a model in which the autonomic nervous system comprised a category. Finally, we performed a structural equation modeling (SEM) analysis to calculate the standardization coefficient and correlation coefficient for each factor.SEM is a statistical model that extends factor analysis and multiple regression analysis. SEM is a statistical approach to understand social phenomena and natural phenomena by introducing latent variables that cannot be observed directly, and then identifying the putative 
causal relationships between latent variables and observed variables. Therefore, it is possible to analyze the relationship between variables without providing a control group. We assessed the data from the model for fitness with the goodness of fit index (GFI), adjusted goodness of fit index (AGFI), and root-mean-square error of approximation (RMSEA) [11] [12]. Analyses were performed using the Statistical Package for the Social Sciences (SPSS), version 25 (SPSS, Inc., Chicago, IL, USA) and SEM was performed using SPSS Amos 19.0 (SPSS, Inc.). Kaiser-Meyer-Olkin (KMO) and Bartlett's tests were performed to determine whether the factor analysis using 10 variables was suitable. The level of significance was set at a p-value of less than $5 \%$.

\section{Results}

\subsection{Main Characteristics}

Among the 42 subjects who agreed to undergo measurements, 35 subjects (mean age, $85.4 \pm 6.1$ years; nine men, 26 women) were included. The main characteristics of the subjects are shown in Table 1 . The average heart rate was $72.9 \pm$ 10.7 beats per minute (bpm), LnTP was $7.40 \pm 1.37$, LnVLF was $6.13 \pm 0.27$, LnLF was $5.47 \pm 2.31$, LnHF was $5.48 \pm 2.55$, LnLF/HF was $1.05 \pm 0.31$, and the average NPI score was $8.4 \pm 9.8$ points, which is considered to be mild BPSD.

\subsection{Results from the Factor Analysis}

The KMO test score was 0.652 and was judged to be a desirable value as a sample. In addition, the p-values of Bartlett's test indicating the correlation between variables rejected the null hypothesis at $p<0.005$. These results showed that the factor analysis using 10 variables was suitable. Therefore, the 10 variables scored were analyzed in the factor analysis. The factor analysis of the 10 variables scored in the 35 participants resulted in several factors with eigenvalues exceeding 1.0. We will first present the three-factor solution (Table 2).

Table 1. Participant characteristics $(\mathrm{n}=35)$.

\begin{tabular}{cc} 
Mean Age & $84.5 \pm 6.1$ \\
Sex & 9 men, 26 women \\
Heart Rate (HR) & $72.9 \pm 10.7$ \\
Total Power (TP) & $7.40 \pm 1.37$ \\
Very Low Frequency (VLF) & $6.13 \pm 0.27$ \\
Low Frequency (LF) & $5.47 \pm 2.31$ \\
High Frequency (HF) & $5.48 \pm 2.55$ \\
LF/HF & $1.05 \pm 0.31$ \\
BPSD (NPI) & $8.4 \pm 9.8$ \\
\hline
\end{tabular}

Total Power (TP): Autonomic nervous system activity; Very Low Frequency (VLF): sympathetic nervous system activity; Low Frequency (LF): sympathetic/parasympathetic nervous system activity; High Frequency (HF): parasympathetic nervous system activity; LF/HF: Autonomic nervous system balance; BPSD: Behavioral and psychological symptoms of dementia. 
Table 2. Results of the factor analysis.

\begin{tabular}{|c|c|c|c|}
\hline & Behavioral state & Mental state & Euphoria \\
\hline Aberrant motor behavior & 0.899 & & \\
\hline Anxiety & 0.867 & & \\
\hline Nighttime behavior disturbances & 0.534 & & \\
\hline Dysphoria/depression & & 0.799 & \\
\hline Agitation/aggression & & 0.716 & \\
\hline Apathy/indifference & 0.49 & 0.561 & \\
\hline Hallucination & & & 0.999 \\
\hline Euphoria/elation & & & 0.455 \\
\hline \multicolumn{4}{|c|}{ The factor correlation matrix contains: } \\
\hline & Behavioral state & Mental state & Euphoria \\
\hline Behavioral state & 1 & 0.221 & -0.037 \\
\hline Mental state & 0.221 & 1 & -0.085 \\
\hline Euphoria & -0.037 & -0.085 & 1 \\
\hline \multicolumn{3}{|c|}{ Kayser-Meyer-Olkin measure of sampling adequacy } & 0.652 \\
\hline Bartlett's test & & p-value & 0.000 \\
\hline
\end{tabular}

Bold indicates a factor loading $>0.4$.

The first factor shows high factor loading for the following four items: "aberrant motor behavior", "anxiety", "nighttime behavior disturbances", and "apathy/ indifference" (absolute factor loading value $\geq 0.4$ ). These four items indicate "behavior state". Thus, factor 1 agrees with the "behavior state". Factor 2 shows high factor loading for two items: "dysphoria/depression", "agitation/aggression", and "apathy/indifference". Thus, the structure of the second factor agrees with the "mental state". Factor 3 shows high factor loading for two items: "hallucination" and "euphoria/elation". However, "hallucination" was excluded from the analysis items because it was not applicable to all subjects, and the third factor was interpreted as "euphoria/elation".

\subsection{Results from the SEM Analysis}

A factor analysis of the nine variables scored from the 35 participants showed a three-factor solution. Therefore, these factors were used to construct a model in which a category affected BPSD. We analyzed this model using SEM. A multiple indicator multiple cause model (MIMIC model) was adopted as an analysis model.

The p-value for the model fitting the chi-squared value (=26.515) was 0.381 and the GFI, AGFI, and RMSEA were $0.872,0.796$, and 0.042 , respectively. Thus, all three fitness statistics indicated a good fit with the overall model. The final estimated model with standardized path coefficients is presented in Figure 1. 


\section{Discussion}

Humans cope with stressful stimuli by creating a "fight or flight" response, such as an increase in heart rate, via sympathetic dominance of autonomic nervous system activity [13]. However, the results of this study suggest in patients with dementia and BPSD, parasympathetic-dominant autonomic nervous system activity opposite that seen in stress-coping mechanisms was present. Brain dysfunction in dementia may include a concomitant autonomic neuropathy that may be indicative of erroneous coping mechanisms and an insufficient SAM system reaction. Given the participants' characteristics and average NPI score (8.4), it is likely that patients with mild BPSD were predominant. Thus, it is presumed that less severe dementia causes autonomic dysfunction.

The question of why the influence of BPSD is reflected in parasympathetic activity remains open. Previous studies show interesting research results on stress coping. Behavioral features of the coping style were divided into an active stress response (reacting excitably to stimuli) and a maintenance stress reaction (reacting quietly, like a freezing response to stimuli). Moreover, these behavioral reactions also contrast neuroendocrinologically [14]. Active coping tends to produce dominant

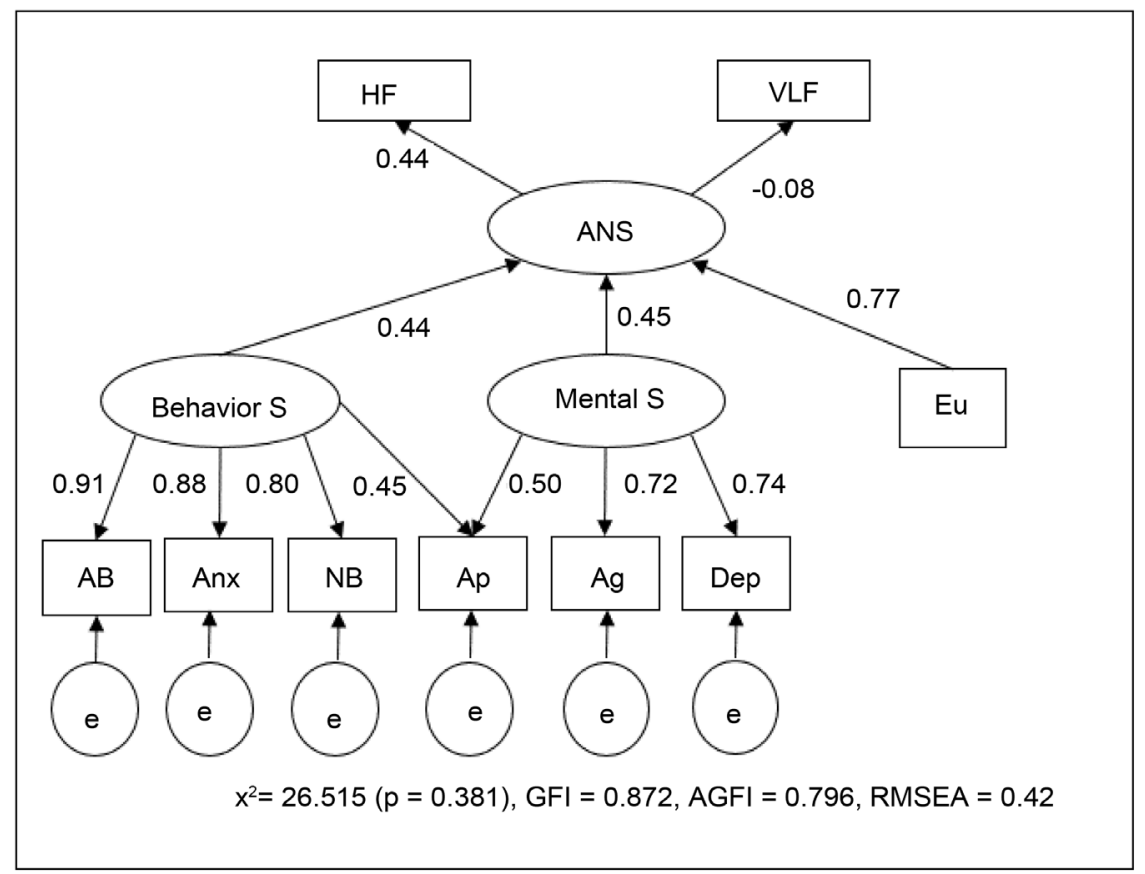

Dep: Dysphoria/depression

AnX: Anxiety

Ap: Apathy/indifference

NB: Nighttime behavior disturbances

VLF: Very low frequency; sympathetic nervous system

HF: High frequency: parasympathetic nervous system

Behavior S: Behavioral State Mental S: Mental state

e: error

Figure 1. Results of the SEM analysis.

\section{Ag: Agitation/aggression \\ Eu: Euphoria/elation \\ $A B$ : Aberrant motor behavior}

ANS: Autonomic nervous system 
sympathetic activity and less HPA system activity, whereas the converse is true for maintenance coping [14] [15].

Based on these previous studies, it is believed that dementia with BPSD occurs in a state of stress, and the subject shows maintenance coping with parasympathetic dominance. It is inferred that a continuation of the state triggers further BPSD and increases the HPA-based reaction in maintenance coping. In this way, stress coping causes not only a reaction to make autonomic nervous system activity sympathetic-dominant but also a reaction causing dominant parasympathetic nervous system activity; the condition then promotes the secretion of anti-stress hormones through the HPA axis.

The response by anti-stress hormones is intended to protect the living body, but canal so have a neurotoxic effect; data indicate it can promote neuronal death in the hippocampus [15]. Therefore, excessive secretion of anti-stress hormone due to chronic stress may not only cause BPSD but also exacerbate cognitive dysfunction in dementia. Accordingly, it is necessary to monitor stress levels and treat potential chronic stress conditions as soon as possible. Autonomic nervous system measurement by heart rate variability analysis, which has a low level of invasiveness and is convenient, may be a useful method. In addition, this study shows that it is necessary to pay attention to not only sympathetic nervous system activity, but also parasympathetic nervous activity when measuring autonomic nervous system activity for stress in dementia. However, such a strategy is viable when brain function is in the normal or near-normal range; if brain function has declined significantly, such a strategy may not be effective.

Finally, the limitations of this research should be noted. In this study, the causal relationship between BPSD and autonomic nervous system activity was analyzed by SEM. However, the collected data is cross-sectional data. An analysis collecting prospective data while observing BPSD would be a useful follow-up.

\section{Conclusion}

This study aimed to clarify the causal relationship between BPSD and autonomic nervous system activity by making a theoretical model in which BPSD influencing autonomic nervous system activity, analyzing the model using SEM, and examining the standardization coefficient. The results showed that the influence of BPSD suppressed sympathetic nervous system activity and enhanced parasympathetic nervous system activity.

\section{Acknowledgements}

This research was supported by JSPS KAKENHI Grant Number C15K01438 (research leader: Daisuke Kimura).

\section{References}

[1] Tible, O.P., Riese, F., Savaskan, E. and von Gunten, A. (2017) Best Practice in the Management of Behavioural and Psychological Symptoms of Dementia. Therapeu- 
tic Advances in Neurological Disorders, 10, 297-309.

https://doi.org/10.1177/1756285617712979

[2] Dodo, N. (2010) Preliminary Examination on Psychoimmunological Index for Patients with Alzheimer's Dementia (AD). Journal of Japanese Society for Dementia Care, 9, 83-88.

[3] Törnhage, C.J. (2009) Salivary Cortisol for Assessment of Hypothalamic-Pituitary-Adrenal Axis Function. Neuroimmunomodulation, 16, 284-287. https://doi.org/10.1159/000216186

[4] Chrous, G.P. and Gold, P.W. (1992) The Concepts of Stress and Stress System Disorders. Overview of Physical and Behavioral Oral Homeostasis. JAMA, 267, 1244-1252. https://doi.org/10.1001/jama.1992.03480090092034

[5] Nakamura, S. (1990) Senile Dementia and Presenile Dementia. The Tohoku Journal of Experimental Medicine, 161, 49-60. https://doi.org/10.1620/tjem.161.Supplement_49

[6] Brody, H. (1976) An Examination of Cerebral Cortex and Brainstem Aging. In: Terry, R.D. and Gershon, S., Eds., Neurobiology of Aging, Raven Press, New York.

[7] Cummings, J.L., Mega, M., Gray, K., Rosenberg-Thompson, S., Carusi, D.A., et al. (1994) The Neuropsychiatric Inventory: Comprehensive Assessment of Psychopathology in Dementia. Neurology, 44, 2308-2314.

https://doi.org/10.1212/WNL.44.12.2308

[8] Task Force of the European Society of Cardiology and the North American Society of Pacing and Electrophysiology (1996) Heart Rate Variability Standards of Measurement, Physiological Interpretation, and Clinical Use. European Heart Journal, 17, 354-381. https://doi.org/10.1093/oxfordjournals.eurheartj.a014868

[9] Gawa, J., Shiwa, S., Nakanishi, D., Kurumaji, M., Kimumoto, O., et al. (2010) On the Autonomic Function Anxiety Using Heart Rate Evaluation of Variability. Japanese Journal of Biofeedback Research, 37, 24-29.

[10] Pett, M.A., Lackey, N.R. and Sullivan, J.J. (2003) Making Sense of Factor Analysis: The Use of Factor Analysis for Instrument Development in Health Care Research. Sage Publications, Inc., Thousand Oaks, CA. https://doi.org/10.4135/9781412984898

[11] Brown, T.A. (2006) Confirmatory Factor Analysis for Applied Research. The Guilford Press, New York.

[12] Browne, M.W. and Cudeck, R. (1993) Alternative Ways of Assessing Model Fit. In: Bollen, K.A. and Long, J.S., Eds. Testing Structural Equation Models, Sage Publications, Inc., Beverley Hills, CA.

[13] Cannon, W.B. (1927) The James-Lange Theory of Emotion: A Critical Examination and Alternative Theory. American Journal of Psychology, 39, 106-124. https://doi.org/10.2307/1415404

[14] Koolhaas, J.M., Korte, S.M., De Boer, S.F., Van Der Vegt, B.J., Van Reenen, C.G., et al. (1999) Coping Styles in Animals; Current Status in Behavior and Stress-Physiology. Neuroscience \& Biobehavioral Reviews, 23, 925-935. https://doi.org/10.1016/S0149-7634(99)00026-3

[15] Koguchi, T. (2001) Research Trend of Stress Coping in Behavioral Medicine. Faculty of Humanities and Social, 52, 69-89. 